\title{
Ultra-relativistic geometrical shock dynamics and vorticity
}

\author{
By JEREMY GOODMA $\mathrm{N}^{1,2} \dagger$ \\ A NDREW MACFADYEN $\mathbf{N}^{1,3}$ \\ ${ }^{1}$ Institute for Advanced Study, Princeton, NJ 08544 \\ ${ }^{2}$ Department of Astrophysical Sciences, Princeton University, Princeton, NJ 08544. \\ ${ }^{3}$ Department of Physics, New York University, New York, NY 10003.
}

(Received ?? and in revised form ??)

Geometrical shock dynamics, also called CCW theory, yields approximate equations for shock propagation in which only the conditions at the shock appear explicitly; the postshock flow is presumed approximately uniform and enters implicitly via a Riemann invariant. The nonrelativistic theory, formulated by G. B. Whitham and others, matches many experimental results surprisingly well. Motivated by astrophysical applications, we adapt the theory to ultra-relativistic shocks advancing into an ideal fluid whose pressure is negligible ahead of the shock, but one third of its proper energy density behind the shock. Exact results are recovered for some self-similar cylindrical and spherical shocks with power-law pre-shock density profiles. Comparison is made with numerical solutions of the full hydrodynamic equations. We review relativistic vorticity and circulation. In an ultrarelativistic ideal fluid, circulation can be defined so that it changes only at shocks, notwithstanding entropy gradients in smooth parts of the flow.

Gamma-ray-burst afterglows have spurred us to look into this problem. These cosmologically distant events are believed to involve a shock launched by the death of a massive star with initial Lorentz factor $\Gamma_{0}>10^{2}$ relative to a pre-shock circumstellar wind (mass density $\left.\rho_{0} \propto r^{-2}\right)$ or interstellar medium ( $\rho_{0} \sim$ constant): see van Paradijs et al. (2000); Piran (2005); Mészáros (2006) for reviews. Light curves fluctuate strongly at early times, probably because of unsteadiness in the source; later, brightness falls approximately as a power law in time but often with undulations that may be due to inhomogeneities ahead of the shock. The observed radiation appears to be synchrotron emission, which implies that $\gtrsim 10^{-2}$ of the postshock energy density takes the form of magnetic field and highly relativistic electrons. Even after compression by the shock, typical circumstellar or interstellar fields would be many orders of magnitude too small. Therefore, it is often supposed that magnetic energy is created rapidly by plasma instabilities at the shock front (Medvedev and Loeb 1999). We wish to explore whether the compressed preshock field might instead be amplified gradually by macroscopic fluid turbulence. The source of the turbulence is supposed to be vorticity produced as the shock passes over inhomogeneities in the ambient medium. Astrophysical applications, however, are deferred to a later paper. Our purpose here is to develop and test suitable theoretical tools: a relativistic version of geometric shock dynamics (hereafter GSD); and, independently, a suitable redefinition of relativistic vorticity that leads to conservation of the circulation on any fluid contour that does not cross a shock.

The elements of nonrelativistic GSD were developed in the 1950s (Moeckel 1952;

$\dagger$ Present address: Princeton University Observatory, Princeton, NJ 08544, U.S.A. e-mail: jeremy@astro.princeton.edu 
Chester 1954; Chisnell 1957; Whitham 1957, 1958, 1959). Whitham (1974) gives a pedagogical review, upon which we have relied heavily. The one-dimensional version of the theory gives a functional or even algebraic relationship (rather than a partial differential equation) between variations in the pre-shock density and variations in the shock Mach number. The multidimensional version describes the effect of changes in shock areadivergence or convergence of the shock normals - on the Mach number. Thus, the theory reduces the dimensionality of the problem by one: in three dimensions, for example, it gives a closed set of equations for the evolution of the shock surface. GSD has even been adapted to reacting flows (detonation waves: Li and Ben-dor 1998).

Naturally, there is a price to be paid in accuracy for these simplifications. For a recent critique, see Baskar and Prasad (2005). Nevertheless, GSD often performs remarkably well when there is reason to expect that fluid gradients or geometrical constraints near the shock ought to dominate, rather than reflections from boundaries behind the shock, and even in some cases where there is no such expectation. GSD successfully describes diffraction of shocks around corners, acceleration of converging shocks, and even the propagation of kinks ("shock shocks") along shock fronts, as judged by comparisons with experiment and with exact self-similar solutions (Bryson and Gross 1961; Schwendeman 1988; Whitham 1974, and references therein).

Relativistic units in which the speed of light $c=1$ will be used. We adopt the conventions of Schutz (1990) for tensors; in particular, the metric in Minkowski coordinates $x^{\mu}=\left(x^{0}=t, x^{1}, x^{2}, x^{3}\right)$ is $\eta_{\mu \nu}=\operatorname{diag}(-1,1,1,1)$, while $T^{\mu \nu}$ and $T^{\bar{\mu} \bar{\nu}}$ denote the components of the same tensor in two Lorentz frames $\mathcal{O}, \overline{\mathcal{O}}$. In all cases considered here, the energy density of the pre-shock fluid will dominated by rest mass, so that pressure and turbulent motions can be neglected ahead of the shock.

\section{Planar shocks}

Our goal is to transcribe GSD for an ultrarelativistic ideal fluid. Following Whitham (1974), we begin with the case that the area of the shock is constant and the pre-shock density $\left(\rho_{0}\right)$ is stratified on planes parallel to the shock front. In place of Mach number, we will be concerned with shock Lorentz factor $(\Gamma)$ or rapidity parameter $(\Phi)$, the two being related by $\Gamma \equiv \cosh \Phi \approx e^{\Phi} / 2 \gg 1$. These quantities are defined in the rest-frame of the pre-shock medium.

The construction of GSD proceeds in two parts. First, the jump conditions are derived from the basic conservation laws; these relate the post-shock fluid properties to the preshock ones if $\Gamma$ is given. This step is potentially exact but simplifies after approximations based on $\Gamma \gg 1, \rho \approx 3 P$, and $P_{0} \ll \rho_{0}$. Next, characteristic equations are derived for the post-shock flow, and the (uncontrolled) approximation is made that one of the Riemann invariants has a known and uniform value behind the shock.

\subsection{Jump conditions}

For a planar shock propagating in the $x^{1}$ direction, the relevant components of the energy-momentum tensor are

$$
T^{00}=(\rho+P) \gamma^{2}-P, \quad T^{01}=(\rho+P) \gamma^{2} \beta, \quad T^{11}=(\rho+P) \gamma^{2} \beta^{2}+P,
$$

where the fluid 4 -velocity has components

$$
U^{\mu} \rightarrow(\gamma, \gamma \beta, 0,0) \equiv(\cosh \phi, \sinh \phi, 0,0)
$$

measured in the shock rest frame. The proper energy density $\rho$ and pressure $P$ are defined in the local fluid rest frame, so that they are Lorentz invariants. 
The jump conditions in the shock rest frame are that $T^{01}$ and $T^{11}$ should be continuous. Since $P \approx \rho / 3$ behind the shock and $P_{0} \ll \rho_{0}$ in front of it, these conditions become

$$
T^{01}: 4 P \gamma^{2} \beta=-\rho_{0} \Gamma^{2} \quad T^{11}: 4 P \gamma^{2} \beta^{2}+P=\rho_{0} \Gamma^{2} .
$$

Consistent with the ultra-relativistic approximation, the pre-shock three-velocity has been set to -1 , which incurs an error $\sim O\left(\Gamma^{-2}\right)$. Eliminating $\Gamma^{2} \rho_{0}$ between the two equations, dividing through by $P$, and setting $\gamma^{-2} \rightarrow 1-\beta^{2}$ yields $(3 \beta+1)(\beta+1)=0$. The root $\beta=-1$ corresponds to no shock at all. Therefore, $\beta=-1 / 3$ in the shock frame. In terms of the rapidity parameters of the fluid and the shock, $\tanh (\Phi-\phi)=1 / 3$. The latter is a covariant formulation since a Lorentz boost along $x^{1}$ with velocity $v$ simply adds $\tanh ^{-1} v$ to both $\phi$ and $\Phi$. Substituting $\beta=-1 / 3$ and $\gamma^{2}=9 / 8$ into either of eqs. (1.2) yields $P=(2 / 3) \Gamma^{2} \rho_{0}$. So, the jump conditions are

$$
\begin{aligned}
& \phi \quad=\Phi-\tanh ^{-1} \frac{1}{3}=\Phi-\ln \sqrt{2}, \\
& \zeta \equiv \frac{\sqrt{3}}{4} \ln P \approx \frac{\sqrt{3}}{4}\left(\ln \rho_{0}+2 \Phi-\ln 6\right) .
\end{aligned}
$$

The peculiar factor $\sqrt{3} / 4$ will simplify the characteristic equations below.

\subsection{Whitham's Characteristic Rule}

Equations (1.3) give two relations among the four variables $\left(\phi, \Phi, \zeta, \ln \rho_{0}\right)$, or equivalently, $\left(\gamma, \Gamma, P, \rho_{0}\right)$. They are exact up to terms $O\left(\Gamma^{-2}\right)$. Whitham's formulation of GSD adds one more condition: the Riemann invariant associated with the characteristics that go upstream from the postshock flow toward the shock, $R_{+}$, is supposed to have the same value as it would if the shock were a transition between constant states. The rationale is that the perturbations to the shock front are supposed to be localized; they are driven by small-scale density variations in the pre-shock fluid, or by local geometrical constraints on the shock, and therefore these perturbations are supposed to average out downstream.

So the next step is to derive the Riemann characteristics from the equations of motion $T_{, \nu}^{\mu \nu}=0$. This has been done in greater generality elsewhere (Martí and Müller 1994), but for completeness we shall rederive the special case we need. With eqs. (1.1), the equations of motion become

$$
\begin{aligned}
& T_{, \nu}^{0 \nu} \propto(2 \cosh 2 \phi+1) \frac{4}{\sqrt{3}} \dot{\zeta}+(4 \sinh 2 \phi) \dot{\phi}+(2 \sinh 2 \phi) \frac{4}{\sqrt{3}} \zeta^{\prime}+(4 \cosh 2 \phi) \phi^{\prime}=0, \\
& T^{1 \nu}{ }_{, \nu} \propto(2 \sinh 2 \phi) \frac{4}{\sqrt{3}} \dot{\zeta}+(4 \cosh 2 \phi) \dot{\phi}+(2 \cosh 2 \phi-1) \frac{4}{\sqrt{3}} \zeta^{\prime}+(4 \sinh 2 \phi) \phi^{\prime}=0,
\end{aligned}
$$

in which the dots denote $\partial / \partial x^{0}$ and the primes $\partial / \partial x^{1}$. Rather than manipulate these equations directly, it is easier to boost into the local rest frame where $\phi=0$, find the characteristics there, and then boost back. Since $\cosh 2 \phi \rightarrow 1$ and $\sinh 2 \phi \rightarrow 0$, the equations above reduce to

$$
\dot{\zeta}+\frac{1}{\sqrt{3}} \phi^{\prime}=0, \quad \dot{\phi}+\frac{1}{\sqrt{3}} \zeta^{\prime}=0 .
$$

By adding and subtracting these, one sees that the characteristic velocities are $\pm 1 / \sqrt{3}$, and the corresponding invariants $\zeta \pm \phi$. Boosting along $x^{1}$ to any other frame simply adds a constant to $\phi$. Therefore,

$$
\begin{aligned}
& R_{+} \equiv \zeta+\phi \text { is constant on } C_{+}:\left(\frac{d x}{d t}\right)_{+}=\tanh \left(\phi+\tanh ^{-1} \frac{1}{\sqrt{3}}\right), \\
& R_{-} \equiv \zeta-\phi \text { is constant on } C_{-}:\left(\frac{d x}{d t}\right)_{-}=\tanh \left(\phi-\tanh ^{-1} \frac{1}{\sqrt{3}}\right) .
\end{aligned}
$$


In Whitham's approximation, $R_{+}$is constant not only along the $C_{+}$characteristics but everywhere in the postshock flow, even immediately behind the shock. Therefore, evaluating $\phi$ and $\zeta$ from the jump conditions (1.3), one obtains

$$
\Phi+\lambda \ln \rho_{0} \approx \text { constant }, \quad \text { where } \lambda \equiv \sqrt{3}-\frac{3}{2} \approx 0.232
$$

This approximate equation predicts how the shock speed slows in response to a transitory increase in pre-shock density: $\Gamma \propto \rho_{0}^{-\lambda}$. With eqs. (1.3), we get the corresponding changes in postshock rapidity and pressure:

$$
\phi+\lambda \ln \rho_{0}=\text { constant; } \quad P \propto \rho_{0}^{1-2 \lambda} .
$$

\subsection{Comparison with an exact self-similar solution}

To reiterate, the approximation (1.5) is intended to describe localized and transitory fluctuations in the propagation of a shock that has some prescribed average Lorentz factor $\bar{\Gamma}$ and advances into a "cold" medium with some prescribed, but spatially variable, pre-shock density $\rho_{0}$ and negligible internal motions and pressure. Whitham (1974) shows that the original nonrelativistic version of his theory approximates rather well the selfsimilar propagation of a planar shock from $x<0$ into a power-law density profile $\rho \propto$ $(-x)^{n}$, even though this situation does not entirely satisfy the assumptions of GSD.

The corresponding ultra-relativistic solution has been given by Sari (2006). In Sari's terminology, the case of interest is a planar (dimensionality parameter $\alpha=0$ ) "Type II" shock with density exponent $k=-n<0$. The Type I (II) solutions are those in which the powerlaw scaling of shock position with time can (cannot) be deduced from global energy conservation. Type II, where the scalings are determined by local conditions near the shock - rather than the inertia of the "piston" behind it - is the case for which one might hope that Whitham's theory would have some success. Indeed, Sari's equation (26) shows that the shock evolves as $\Gamma \propto \rho^{-\lambda}$ with $\lambda$ exactly as in eq. (1.5)! It is not clear why the agreement should be exact, but presumably the extreme simplicity of the ultrarelativistic fluid equations is somehow responsible.

\section{Non-planar shocks}

No vorticity can be created by an exactly planar shock, yet the 1D theory above may be adequate for estimating the vorticity produced by encounters between an ultrarelativistic shock and a density inhomogeneity. Lorentz contraction causes the lateral dimension of inhomogeneities viewed in the shock or postshock frame to be larger by a factor $\Gamma \gg 1$ than the longitudinal ones, so that changes in speed and pressure are impressed upon the immediately postshock flow before it "notices" that the changes differ at other lateral positions. Thus it should usually be sufficient to evaluate the flow changes from the $1 \mathrm{D}$ theory, and then take lateral derivatives to evaluate the resulting vorticity.

Nevertheless, it is worthwhile to extend the ultrarelativistic version of Whitham's theory to nonplanar shocks for several reasons:

- in order to study the stability of the shock;

- in order to compare with exact spherical and cylindrical self-similar solutions, and with numerical tests such as refraction around an (oblique) corner;

- because the extension is not difficult.

The idea of Whitham's nonplanar extension is to insert a factor representing changes in shock area into the conservative form of the fluid equations. Thus let $x^{1}$ be a coordinate measuring arc length along the shock normal, and $x^{2}$ and $x^{3}$ be coordinates in the shock 
surface defined in such a way that a point moving along the shock normal maintains constant $\left(x^{2}, x^{3}\right)$. The equations of motion are taken to be

$$
\begin{aligned}
& T^{00}{ }_{, 0}+A^{-1}\left(A T^{01}\right)_{, 1}=0 \\
& T^{10}{ }_{, 0}+A^{-1}\left(A T^{11}\right)_{, 1}=0 .
\end{aligned}
$$

Here $A$ is the 2D Jacobian relating the area of an element of the shock surface to its initial area. More precisely, since the characteristic equation does not hold across the shock, $A$ represents the cross-sectional area of a bundle of streamlines immediately behind the shock; since the pre-shock medium is assumed to be at rest, the flow behind the shock is normal to it.

Equations (2.1) are not fully equivalent to $T_{; \nu}^{\mu \nu}=0$ : for $\mu=1$, they do not contain the part of the covariant derivative associated with turning of the shock normal. They do however represent the divergence or convergence of the normals, which leads to area change and strengthens or weakens the shock. Because of the terms involving $A$, the quantities $R_{ \pm}$are no longer invariant along their respective characteristics $C_{ \pm}$. The equation for $R_{+}$works out to

$$
\begin{aligned}
\left(\frac{\mathrm{d}}{\mathrm{d} s}\right)_{+}(\zeta+\phi) & =-\frac{\sinh \phi}{\sqrt{3} \sinh \phi+\cosh \phi}\left(\frac{\mathrm{d}}{\mathrm{d} s}\right)_{+} \ln A \\
& \rightarrow-\frac{1}{\sqrt{3}+1}\left(\frac{\mathrm{d}}{\mathrm{d} s}\right)_{+} \ln A,
\end{aligned}
$$

where $(\mathrm{d} / \mathrm{d} s)_{+} \equiv \partial_{1}+\left(v_{+}\right)^{-1} \partial_{1}$ is the derivative along the $C_{+}$characteristic, and $v_{+} \equiv$ $\tanh \left[\phi+\tanh ^{-1}(1 / \sqrt{3})\right]$ is the characteristic velocity. The final form of eq. (2.2) is in the pre-shock rest frame where $\cosh \phi \approx \sinh \phi \approx 1$ up to $O\left(\Gamma^{-2}\right)$.

The jump conditions (1.3) are unchanged. Inserting these into (2.2) yields

$$
\begin{aligned}
& \frac{\mathrm{d}}{\mathrm{d} s}\left(\Phi+\lambda \ln \rho_{0}+\mu \ln A\right) \approx 0, \\
& \lambda \equiv \sqrt{3}-\frac{3}{2}, \quad \mu \equiv 3 \sqrt{3}-5 .
\end{aligned}
$$

Following Whitham, we have made the approximation that the characteristic equation applies on the shock, although its propagation speed [rapidity $\Phi=\phi+\tanh ^{-1}(1 / 3)$ ] is not quite the same as that of the characteristic $\left[\tanh ^{-1} v_{+}=\phi+\tanh ^{-1}(1 / \sqrt{3})\right]$ Consistent with this approximation, the derivative $\mathrm{d} / \mathrm{d} s$ in (2.3) is taken to be the derivative with respect to arc length along the shock normal. Equation (2.3) predicts that the shock decelerates locally where its area increases, and accelerates where the area decreases. This will tend to stabilize corrugations in the shock front.

Equations (2.3) need to be supplemented by a vector equation for the shock normals. Introduce a function $\tau(x, y, z)$ such that the locus of the shock in Minkowski coordinates is described by $t=\tau(x, y, z)$. The normal to the shock is then $\vec{n}=\vec{\nabla} \tau /|\vec{\nabla} \tau|$, and its 3 -velocity is $\vec{V}=\vec{n} /|\vec{\nabla} \tau|$. The area function $A$ of the shock satisfies

$$
\vec{\nabla} \cdot\left(\frac{\vec{n}}{A}\right)=0 \text {. }
$$

This is a purely geometrical, rather than dynamical, statement. Whitham (1974) demonstrates it by applying Gauss's Law to a "flux tube" whose sides are made up of integral curves of $\vec{n}$, and whose ends are elements of the shock surface at different times. 


\subsection{Comparison with nonplanar self-similar solutions}

Sari (2006)'s equation (26) for Type II solutions is equivalent to

$$
\frac{\mathrm{d}}{\mathrm{d} r} \ln \Gamma=\alpha(5-3 \sqrt{3})-\left(\sqrt{3}-\frac{3}{2}\right) \frac{\mathrm{d}}{\mathrm{d} r} \ln \rho,
$$

$\rho \propto r^{-k}$ being the pre-shock density. Here $\alpha=0,1,2$ for planar, cylindrical, and spherical shocks, respectively. Since the area factor should scale as $A \propto r^{\alpha}$ in these three geometries, equation (2.3) predicts Sari's result perfectly.

In these self-similar solutions, dimensionality plays a limited role since individual shock normals are constant. Whitham (1974) discusses applications of the nonrelativistic theory to non-self-similar and truly multidimensional problems such as refraction of shocks around corners and obstacles. Here we can expect eqs. (2.3 \& (2.4) not to be exact since, as noted above, they do not incorporate the full covariant derivatives in the equations of motion, and since the streamlines behind the shock are not perfectly straight.

\subsection{Detailed treatment of initially planar shocks in two dimensions.}

These details will facilitate comparison with numerical solutions of the full hydrodynamic equations for two-dimensional test cases (\$4). They also serve to illustrate effects that involve changes in the shock normal, including the transverse propagation of disturbances along the shock front.

We take $z$ to be the ignorable coordinate. Following Whitham again, let $\psi$ be the angle between the normal and the $x$ axis, so that $\partial \tau / \partial x=|\vec{\nabla} \tau| \cos \psi=V^{-1} \cos \psi$ and $\partial \tau / \partial y=V^{-1} \sin \psi$. Equation (2.4) can then be rephrased as the two first-order equations

$$
\begin{aligned}
\frac{\partial}{\partial x}\left(V^{-1} \sin \psi\right)-\frac{\partial}{\partial y}\left(V^{-1} \cos \psi\right) & =0, \\
\frac{\partial}{\partial x}\left(A^{-1} \cos \psi\right)+\frac{\partial}{\partial y}\left(A^{-1} \sin \psi\right) & =0,
\end{aligned}
$$

of which the first is simply the statement that $\partial^{2} \tau / \partial y \partial x=\partial^{2} \tau / \partial x \partial y$. Together with (2.3), equations (2.5) form a hyperbolic system. This is especially clear in the paraxial approximation where $\psi \sim O\left(\Gamma^{-2}\right)$. To this order, we may then replace $\sin \psi \rightarrow \psi$, $\cos \psi \rightarrow 1$, and $V \rightarrow 1-\left(2 \Gamma^{2}\right)^{-1}$, so that eqs. (2.5) become

$$
\begin{aligned}
\frac{\partial \psi}{\partial x}+\frac{1}{2} \frac{\partial}{\partial y}\left(\psi^{2}-\Gamma^{-2}\right) & =0, \\
\frac{\partial}{\partial x} A^{-1}+\frac{\partial}{\partial y}\left(A^{-1} \psi\right) & =0 .
\end{aligned}
$$

With our ordering, the term in $\psi^{2}$ is of higher order than the others - it results from taking $\cos \psi=1-\psi^{2} / 2$ rather than unity in the first of eqs. (2.6) - but it does no harm and in fact makes the characteristic velocities work out more neatly.

If the shock is initially planar and the pre-shock density initially uniform, then (2.3) implies that $\Phi+\lambda \ln \rho_{0}+\mu \ln A$ is constant throughout the flow. Thus $\Gamma$ in the first of equations (2.6) is to be regarded as a function of $A$ and $(x, y)$, given by

$$
\Gamma(A, x, y)=\bar{\Gamma} \bar{\rho}^{\lambda} \bar{A}^{\mu} \rho_{0}^{-\lambda} A^{-\mu},
$$

in which the barred quantities are constants pertaining to the initially uniform medium and planar shock, and $\rho_{0}(x, y)$ is a prescribed function. The characteristic velocities of 
the system (2.6)-(2.7) are

$$
\left(\frac{d y}{d x}\right)_{ \pm}=\psi \pm \mu^{1 / 2} \Gamma^{-1}
$$

The factor of $\Gamma^{-1}$ is easy to interpret as a consequence of relativistic beaming. A disturbance propagating at the speed of light along an otherwise planar shock front would have transverse velocity $\mathrm{d} y / \mathrm{d} t= \pm \Gamma^{-1}$ in the pre-shock rest frame. To leading order in $\Gamma^{-1}$, we may replace $\mathrm{d} t$ with $\mathrm{d} x$ in this expression, so with $\mu^{1 / 2} \approx 0.4429$, the characteristics (2.8) are subluminal.

Since eqs. (2.5) and (2.6) are in conservation form, we may use them to study discontinuities in the shock front itself: "shock shocks." For a shock shock propagating at slope $U \equiv(d y / d x)_{\mathrm{ss}}$, the jump conditions implied by (2.6) are

$$
\begin{aligned}
{\left[-2 U \psi+\psi^{2}-\Gamma^{-2}\right] } & =0, \\
{\left[A^{-1}(\psi-U)\right] } & =0,
\end{aligned}
$$

where $[Q]$ denotes the discontinuity in quantity $Q$ across the shock shock. Let us assume a homogenous pre-shock medium, $\rho_{0}=\bar{\rho}=$ constant, $\Gamma=$ constant. Then if $\psi=0$ and $A=1$ ahead of the shock shock, the post-shock-shock quantities $\left(\psi^{\prime}, A^{\prime}\right)$ satisfy

$$
(U \Gamma)^{2}=\frac{\left(A^{\prime}\right)^{2 \mu}-1}{\left(A^{\prime}\right)^{2}-1}, \quad \psi^{\prime}=U\left(1-A^{\prime}\right) .
$$

Thus in the limit $A^{\prime} \rightarrow 0$, we have $\psi^{\prime}=U=1 / \Gamma$, and it follows from eq. (2.7) that $\Gamma^{\prime} \rightarrow \infty$. In the opposite limit $A^{\prime} \gg 1$-but still $A^{\prime} \ll \Gamma^{1 / \mu}$ so that $\Gamma^{\prime} \gg 1$ (else the ultrarelativisitic approximation would not apply) - we have $\psi^{\prime}=-\left(A^{\prime}\right)^{\mu} / \Gamma=-1 / \Gamma^{\prime}$ and $U=-\psi^{\prime} / \Gamma^{\prime}$.

Finally, because gamma-ray-burst shocks are believed to emanate from effectively pointlike explosions, it is of interest to consider a nearly spherical rather than planar shock. This case is effectively two-dimensional if the perturbations are axisymmetric. We take polar coordinates $(r, \theta, \phi)$ such that $\theta=0, \pi$ is the axis of symmetry and define $\psi$ to be the angle between the normal and radial directions, i.e. $\vec{n} \cdot \vec{r}=\cos \psi$. The analogs of eqs. (2.5) then become

$$
\begin{aligned}
\frac{\partial}{\partial r}\left(\frac{r \sin \psi}{V}\right)-\frac{\partial}{\partial \theta}\left(\frac{\cos \psi}{V}\right) & =0, \\
\frac{\partial}{\partial r}\left(\frac{r^{2} \cos \psi}{A}\right)+\frac{r}{\sin \theta} \frac{\partial}{\partial \theta}\left(\frac{\sin \theta \sin \psi}{A}\right) & =0,
\end{aligned}
$$

and for $\psi \ll 1, \Gamma \gg 1$, eqs. (2.6) become

$$
\begin{aligned}
\frac{\partial}{\partial r}(r \psi)+\frac{1}{2} \frac{\partial}{\partial \theta}\left(\psi^{2}-\Gamma^{-2}\right) & =0, \\
\frac{\partial}{\partial r}\left(\frac{r^{2}}{A}\right)+\frac{r}{\sin \theta} \frac{\partial}{\partial \theta}\left(\frac{\psi \sin \theta}{A}\right) & =0,
\end{aligned}
$$

while (2.7) is unchanged.

\section{Relativistic vorticity}

This discussion in this section is independent of the approximations of GSD, although the ultrarelativistic equation of state $P=\rho / 3$ figures prominently. 
As explained above, we are motivated by the need to explain the amplification of magnetic field behind the shocks associated with gamma-ray bursts, and by the possible role of turbulence in this amplification. Therefore, it may be worthwhile to record our assumptions about the relation of post-shock vorticity to the magnetic field.

It follows from the induction equation of ideal magnetohydrodynamics,

$$
\partial_{t} \vec{B}=\vec{\nabla} \times(\vec{v} \times \vec{B})
$$

that magnetic energy increases according to

$$
\frac{\mathrm{d}}{\mathrm{d} t} \int \vec{B} \cdot \vec{B} \mathrm{~d}^{3} \vec{x}=\int B_{i} B_{j} \partial_{i} v_{j} \mathrm{~d}^{3} \vec{x}
$$

which involves the instantaneous shear $\left(\partial_{i} v_{j}+\partial_{j} v_{i}-\frac{2}{3} \vec{\nabla} \cdot \vec{v}\right)$ and convergence $(\vec{\nabla} \cdot \vec{v})$ of the velocity field rather than the vorticity, which is its curl. Nevertheless, vorticity is important to secular amplification of the field by localized disturbances. In an ideal fluid, a localized nonvortical disturbance evolves into sound waves, whose oscillations produce only transitory changes in magnetic energy, and which propagate away from their source. Energy in vortical motions, however, remains localized, and the shear between neighboring eddies is expected to amplify the field exponentially on their turnover time.

\subsection{Vorticity and circulation}

Non-relativistically, the vorticity $\vec{\omega} \equiv \vec{\nabla} \times \vec{v}$, where $\vec{v}$ is the fluid three-velocity. In a compressible but isentropic fluid without shocks, Kelvin's Circulation theorem is

$$
\frac{\mathrm{d}}{\mathrm{d} t} \oint_{C} \vec{v} \cdot d \vec{l}=0,
$$

where $C$ is closed contour advected by the flow.

The generalization of $\vec{\omega}$ and eq. (3.2) to relativistic flow is not entirely straightforward (Eshraghi 2003). Let $\vec{U}=\left(U^{0}, U^{1}, U^{2}, U^{3}\right)$ be the 4-velocity of the fluid, so that $\eta_{\mu \nu} U^{\mu} U^{\nu}=-1$. In terms of the local rest-frame energy density $\rho$ and pressure $P$, the energy-momentum tensor is

$$
T^{\mu \nu}=(\rho+P) U^{\mu} U^{\nu}+\eta^{\mu \nu} P .
$$

The equations of motion

$$
T_{, \nu}^{\mu \nu}=0
$$

must be supplemented by an equation of state. Normally this involves two independent thermodynamic variables, e.g. $P=P(\rho, T), P(\rho, N)$, or $P(N, S)$, where $T$ is the restframe temperature, $N$ is the proper number density of conserved particles, and $S$ is the entropy per particle. An essential feature of ultra-relativistic shocks is that the postshock particles are highly relativistic in the fluid rest frame, so that $P=\rho / 3 甘$ To the extent that the fluid is ideal [eq. (3.3)], entropy and temperature gradients then have no effect on the flow- except at shocks, but even there they do not have to be addressed explicitly.

To illustrate this point, we consider a general equation of state in which entropy does influence the dynamics. The conservation of particle number is expressed by

$$
\left(N U^{\mu}\right)_{, \mu}=0 .
$$

$\dagger$ This assumes that the stress is isotropic, which is not at all obvious in astrophysical applications where the plasma is collisionless. 
The vorticity turns out to be best formulated as

$$
\Omega_{\mu \nu} \equiv-H_{\mu, \nu}+H_{\nu, \mu}
$$

in terms of the relativistic enthalpy $h$ and its associated current $H^{\mu}$ :

$$
h \equiv \frac{\rho+P}{N}, \quad H^{\mu} \equiv h U^{\mu} .
$$

With the First Law $d(\rho / N)=T d S-P d(1 / N)$ in the form $n^{-1} d P=d h-T d S$, eq. (3.4) can be rewritten as

$$
U^{\nu} H_{\mu, \nu}=T S_{, \mu}-h_{, \mu} .
$$

The vorticity equation then follows from the "curl" of this, namely

$$
\begin{gathered}
-\left(U^{\nu} H_{\alpha, \nu}\right)_{, \beta}+\left(U^{\nu} H_{\beta, \nu}\right)_{, \alpha}=U^{\nu} \Omega_{\alpha \beta, \nu}+U^{\nu}{ }_{, \beta} \Omega_{\alpha \nu}+U^{\nu}{ }_{, \alpha} \Omega_{\nu \beta} \\
=T, \alpha \\
, \beta
\end{gathered}
$$

The last line above vanishes if the entropy is uniform, $S, \mu=0$, or more generally if there is only one independent thermodynamic quantity so that $S=S(T)$. The intermediate expression is the Lie derivative $\mathcal{L}_{\vec{U}}$ of the vorticity considered as a 2-form,

$$
\boldsymbol{\Omega} \equiv \mathbf{d} \boldsymbol{H} \equiv H_{\nu, \mu} \mathbf{d} \boldsymbol{x}^{\mu} \wedge \mathbf{d} \boldsymbol{x}^{\nu},
$$

with respect to the 4 -velocity $\vec{U}$. The statement of conservation of circulation is then

$$
\frac{\mathrm{d}}{\mathrm{d} \tau} \oint_{\partial A} H_{\alpha} d x^{\alpha}=\mathcal{L}_{\vec{U}} \iint_{A} \boldsymbol{\Omega}=0,
$$

where $A$ is a surface advected by $\vec{U}$ and $\partial A$ is the curve bounding it, and $d / d \tau \equiv U^{\mu} \partial_{\mu}$ is the convective derivative. Since different parts of the fluid move with different Lorentz factors, the surface $A$ and curve $\partial A$ will not (in general) remain within hyperplanes of constant Minkowski time $t$, unfortunately.

Now we specialize to the ultra-relativistic equation of state $P=\rho / 3$. The true entropy per particle is $S \propto \ln \left(P / N^{4 / 3}\right)$, which will not be uniform after the shock passes over density inhomogeneities. However, if we define an ersatz number density $\tilde{N} \propto P^{3 / 4}$ at some initial time, so that $P / \tilde{N}^{4 / 3}$ is spatially uniform, and if we demand that $\tilde{N}$ evolve according to (3.5) with $\tilde{N}$ instead of $N$, then $P / \tilde{N}^{4 / 3}$ will remain uniform in smooth parts of the flow, though not across shocks. This follows because $U_{\mu} T^{\mu \nu}{ }_{, \nu}=$ $-3 U^{\nu} P_{, \nu}-4 P U^{\mu}{ }_{\mu}=0$, whence $U^{\nu}\left(P / \tilde{N}^{4 / 3}\right)_{, \nu}=0$.

We don't actually have to deal with $\tilde{N}$ directly. Since $\tilde{h} \equiv(\rho+P) / \tilde{N}=4 P^{1 / 4}$, we can simply redefine the enthalpy current as

$$
H^{\mu}=P^{1 / 4} U^{\mu} \quad(\text { when } P=\rho / 3 \text { only }) .
$$

Then eq. (3.8) becomes

$$
U^{\nu} H_{\mu, \nu}=-\left(P^{1 / 4}\right)_{, \mu}, \quad P=\left(H^{\mu} H_{\mu}\right)^{2} .
$$

The vorticity defined in terms of this $\vec{H}$ via (3.6) is conserved in the sense that the righthand side of (3.9) vanishes in all smooth parts of the flow, even after shocks. In particular, if $\boldsymbol{\Omega}=0$ initially then it remains zero as long as the flow remains smooth. The jump conditions that follow from integrating (3.4) across shocks are not equivalent to the corresponding integral of (3.9), however, and so this vorticity can be created at shocks. 


\subsection{Vorticity vector}

$\Omega_{\alpha \beta}=-\Omega_{\beta \alpha}$ would appear to have six algebraically independent components. In fact it has only three. To see this, note that

$$
-h_{, \mu}=\left(H_{\nu} U^{\nu}\right)_{, \mu}=H_{\nu, \mu} U^{\nu}+H_{\nu} U^{\nu}{ }_{, \mu}=H_{\nu, \mu} U^{\nu}+h\left(U_{\nu} U^{\nu}\right)_{, \mu}=H_{\nu, \mu} U^{\nu} .
$$

Therefore if the fluid is isentropic, or if it is ultrarelativistic and the enthalpy current is defined by eq. (3.10), then eq. (3.8) becomes

$$
U^{\nu} \Omega_{\mu \nu}=0 .
$$

This means that in the local rest frame, where $U^{\nu} \rightarrow \delta_{0}^{\nu}$, the "electric" components $\Omega_{i 0}=-\Omega_{0 i}$ of the vorticity vanish, and only the three "magnetic" components $\Omega_{i j}=$ $-\Omega_{j i}$ survive. Let $\vec{\omega}$ be the three-vector field with components $\omega_{i}=\epsilon_{i j k} \Omega_{j k} / 2$ in an arbitrary inertial frame (not necessarily coinciding with the local fluid rest frame). If one uses eq. (3.12) to eliminate the inertial components $\Omega_{i 0}$ from the identity

$$
\Omega_{\alpha \beta, \gamma}+\Omega_{\beta \gamma, \alpha}+\Omega_{\gamma \alpha, \beta}=0
$$

which is the tensorial expression of $\mathbf{d}(\mathbf{d} \boldsymbol{H})=0$, the result is

$$
\partial_{t} \vec{\omega}-\vec{\nabla} \times(\vec{v} \times \vec{\omega})=0,
$$

where $\vec{v}$ is the three-velocity, $v^{i}=U^{i} / U^{0}$. This is formally identical to the nonrelativistic vorticity equation of an isentropic fluid, except that $\vec{\omega}$ is $\vec{\nabla} \times \vec{H}$ rather than $\vec{\nabla} \times \vec{v}$. The derivation just given, which parallels that of the induction equation (3.1), shows that eq. (3.13) is relativistically covariant.

\section{Numerical simulations and tests}

In order to test the predictions of the GSD theory we have performed a series of numerical simulations with the the RAM special relativistic hydrodynamics code Zhang and MacFadven (2006). The simulations test the variations of pressure and Lorentz factor behind a strong shock as it passes over density perturbations in the pre-shock medium. The simulations are performed on the domain $x=[0.0,1.0]$ with reflecting boundary at $x=0.0$ and outflow (zero gradient) boundary at $x=1$ and adiabatic index $\gamma_{a}=4 / 3$. In the notation of the present paper, the equation of state is $P=\left(\gamma_{a}-1\right)(\rho-m N)$, where $m$ is the rest mass per particle.

We have performed ten simulations in which the pressure is initially set to $P=10^{5}$ for $x<0.001$ and $P=10^{-6}$ elsewhere, to create a plane-parallel analog of an explosively driven spherical gamma-ray-burst shock. The rest-mass contribution $m N$ to the energy density for $x=[0.8,0.9]$ is initially set to one of the ten perturbed values $\rho_{p}=\{1.0,1.2,1.4,1.6,1.8,2.0,5.0,10.0,20.0,30.0\}$ and to $\rho_{i}=1.0$ elsewhere. The ten simulations are otherwise identical. A strong relativistic shock with Lorentz factor $\Gamma \sim 10$ is initially driven into the medium as the over-pressured region expands and a thin relativistic shell forms behind it. As the shock crosses the (positive) density perturbation, its Lorentz factor decreases and post-shock pressure increases with the values seen in Fig. 1. Note that $\gamma$ is the Lorentz factor of the fluid rather than that of the shock itself; for an ultrarelativistic shock advancing into a cold, stationary medium, the jump condition (1.3a) implies that these two Lorentz factors are represented simply by $\gamma=\Gamma / \sqrt{2}$, a relation that does not depend upon the approximations of GSD. We measure $\gamma$ and $P$ at $t=0.9$, after the shock has crossed the perturbation region and is at $x \approx 0.9$, and we record for that time slice the values $P_{m}$ and $\gamma_{m}$ where the pressure reaches its maximum 

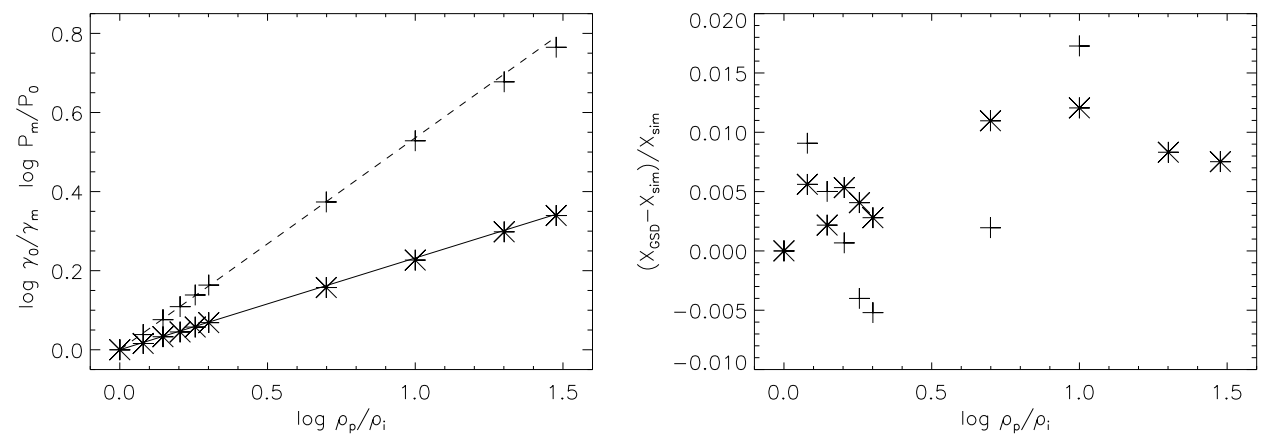

Figure 1. Comparison between analytic GSD predictions and numerical simulations for post-shock Lorentz factor and pressure as a function of the pre-shock density perturbation. The right panel shows the fractional difference between the GSD prediction $\mathrm{X}_{\mathrm{GSD}}$ and the simulation value $\mathrm{X}_{\text {sim }}$, where $\mathrm{X}$ stands for maximum pressure $P_{m}$ (plus signs) or Lorentz factor $\gamma_{m}$ (asterisks).

post-shock value. In Fig. 1 (left panel) we show the logarithms of $P_{m}$ (plus signs) and $\gamma_{m}^{-1}$ (asterisks) versus $\log \left(\rho_{p} / \rho_{i}\right)$. The ordinates have been scaled by the values $P_{\circ}$ and $\gamma_{\circ}$ obtained from a fiducial run with uniform density $\left(\rho_{p}=\rho_{i}=1\right)$. The lines show the scaling predicted by the GSD approximation with slopes $\lambda=\sqrt{3}-\frac{3}{2} \approx 0.232$ (solid) and $1-2 \lambda \approx 0.536$ (dashed). In Fig. 1 (right panel), we show the fractional difference between the GSD prediction and the numerical simulation for maximum pressure (plus signs) and Lorentz factor (asterisks).

We find that the GSD approximation works surprisingly well even for large density perturbations, justifying its use up to density contrasts of factors of ten or more.

We acknowledge the use of the Scheides cluster at the Institute for Advanced Study. The software used in this work was in part developed by the DOE-supported ASCI/Alliance Center for Astrophysical Thermonuclear Flashes at the University of Chicago.

\section{REFERENCES}

S. Baskar and Phoolan Prasad. Propagation of curved shock fronts using shock ray theory and comparison with other theories. J. Fluid Mech., 523:171-198, Jan 2005.

A. E. Bryson and R. W. F. Gross. Diffraction of strong shocks by cones, cylinders, and spheres. J. Fluid Mech., 10:1-16, 1961.

W. Chester. The quasi-cylindrical shock tube. Phil. Mag., 45(7):1293-1301, 1954.

R. F. Chisnell. The motion of a shock wave in a channel, with applications to cylindrical and spherical shock waves. J. Fluid Mech., 2, 1957.

H. Eshraghi. On the vortex dynamics in fully relativistic plasmas. Physics of Plasmas, 10(9): 3577-3583, 2003.

H. Li and G. Ben-dor. Modified CCW theory for detonation waves. Combustion and Flame, 113:1-12, Apr 1998.

J.M. Martí and E. Müller. The analytical solution of the Riemann problem in relativistic hydrodynamics. J. Fluid Mech., 258:317-333, 1994.

M. V. Medvedev and A. Loeb. Generation of Magnetic Fields in the Relativistic Shock of Gamma-Ray Burst Sources. Astrophys. J., 526:697-706, December 1999.

P. Mészáros. Gamma-ray bursts. Reports of Progress in Physics, 69:2259-2321, August 2006. .

W. E. Moeckel. Interaction of oblique shock waves with regions of variable pressure, entropy, and energy. Technical Report 2725, Nat. Adv. Comm. Aero, Washington, 1952.

T. Piran. Magnetic Fields in Gamma-Ray Bursts: A Short Overview. In E. M. de Gouveia dal 
Pino, G. Lugones, and A. Lazarian, editors, AIP Conf. Proc. 784: Magnetic Fields in the Universe: From Laboratory and Stars to Primordial Structures., pages 164-174, September 2005. .

R. Sari. First and second-type self-similar solutions of implosions and explosions containing ultrarelativistic shocks. Physics of Fluids, 18:027106, 2006. .

B. F. Schutz. A first course in general relativity. Cambridge University Press, 1990.

D. W. Schwendeman. Numerical scheme for shock propagation in three dimensions. Proc. Roy. Soc. London Ser. A, 416(1850):179-198, Mar 1988.

J. van Paradijs, C. Kouveliotou, and R. A. M. J. Wijers. Gamma-Ray Burst Afterglows. Ann. Rev. Astron. Astrophys., 38:379-425, 2000. .

G. B. Whitham. A new approach to problems of shock dynamics. Part I. Two-dimensional problems. J. Fluid Mech., 2:146-171, 1957.

G. B. Whitham. On the propagation of shock waves through regions of non-uniform area or flow. J. Fluid Mech., 4:337-360, 1958.

G. B. Whitham. New approach to problems of shock dynamics. Part II. Three-dimensional problems. J. Fluid Mech., 5:369-386, 1959.

G. B. Whitham. Linear and Nonlinear Waves. Wiley, 1974.

W. Zhang and A. I. MacFadyen. RAM: A Relativistic Adaptive Mesh Refinement Hydrodynamics Code. Astrophys. J. Suppl., 164:255-279, May 2006. . 